\title{
Presence and function of microRNA-92a in chondrogenic ATDC5 and adipose-derived mesenchymal stem cells
}

\author{
CHANGHE HOU $^{1 *}$, ZIJI ZHANG $^{1 *}$, ZHIQI ZHANG $^{1}$, PEIHUI WU $^{1}$, XIAOYI ZHAO ${ }^{1}$, \\ MING FU ${ }^{1}$, PUYI SHENG ${ }^{1-3}$, YAN KANG ${ }^{1}$ and WEIMING LIAO ${ }^{1}$ \\ ${ }^{1}$ Department of Joint Surgery, The First Affiliated Hospital of Sun Yat-Sen University, Guangzhou, Guangdong 510080; \\ ${ }^{2}$ Huangpu Joint Centre, The First Affiliated Hospital of Sun Yat-Sen University, Guangzhou, Guangdong 510700; \\ ${ }^{3}$ Institute of Orthopedics, The First Affiliated Hospital of Sun Yat-Sen University, Guangzhou, \\ Guangdong 518000, P.R. China
}

Received September 12, 2014; Accepted May 28, 2015

DOI: $10.3892 / \mathrm{mmr} .2015 .4008$

\begin{abstract}
The aim of the present study was to investigate the presence and biological function of microRNA-92a (miR-92a) in chondrogenesis and cartilage degeneration. Human adipose-derived mesenchymal stem cells (hADSCs) in micromass and chondrocyte-like ATDC5 cells were induced to chondrogenesis, and primary human/mouse chondrocytes (PHCs/PMCs) and chondrogenic ATDC5 cells were stimulated with interleukin-1 $\beta$ (IL-1 $\beta$ ). An miR-92a mimic/inhibitor was transfected into the ATDC5 cells using lipofectamine 2000. Gene expression was analyzed using reverse transcription-quantitative polymerase chain reaction. Alcian blue was used to stain the cartilage nodules and chondrogenic micromass. The potential target genes, signaling pathways and functions of miR-92a were examined using miRanda, miRDB, CLIP-Seq, TargetScan and Kyoto Encyclopedia of Genes and Genomes. The expression of miR-92a was elevated in the chondrogenic ATDC5 cells and hADSCs, and also in the IL-1 $\beta$-induced ATDC5 cells, PMCs and PHCs. Forced expression of miR-92a enhanced the expression levels of col9a2 and aggrecan. A total of 279 genes were predicted as potential target genes of miR-92a. The phosphoinositide 3-kinase/PI3K)-Akt, ErbB and focal adhesion kinase pathways, extracellular matrix (ECM)-receptor interaction and the mammalian target of rapamycin (mTOR) signaling pathway were suggested to mediate the effects of
\end{abstract}

Correspondence to: Dr Weiming Liao or Dr Yan Kang, Department of Joint Surgery, The First Affiliated Hospital of Sun Yat-Sen University, 58 Zhongshan No. 2 Road, Yuexiu, Guangzhou, Guangdong 510080, P.R. China

E-mail: 1wmjoint@163.com

E-mail: neokang@163.com

*Contributed equally

Key words: chondrogenesis, cartilage, osteoarthritis, microRNA-92a, col9a2
miR-92a on chondrogenesis and cartilage degeneration. These results demonstrated that miR-92a was involved in chondrogenesis and the chondrocyte response induced by IL-1 $\beta$. miR-92a positively contributed to the expression of col9a 2 and of aggrecan.

\section{Introduction}

Cartilage tissues are degenerated and are destroyed in osteoarthritic joints, which are more prevalent in elderly individuals (1). Although arthroplasty can efficiently relieve the symptoms of osteoarthritis, implant loosening is inevitable in the years following arthroplasty (1). Tissue engineered cartilage has been suggested as an improved substitution for conventional arthroplasty. Therefore, it is necessary to understand the molecular mechanisms underlying cartilage generation and degeneration.

Subsequent to mesenchymal condensation, mesenchymal stem cells sense cell-cell and cell-extracellular matrix (ECM) contact, which is termed focal adhesion (2), followed by differentiation into chondrocytes and expression of ECM. There are other exogenous stimuli and intracellular signaling pathways regulating chondrogenesis and cartilage degeneration, including the phosphoinositide 3-kinase(PI3K)-Akt, mammalian target of rapamycin (mTOR) and epidermal growth factor pathways (3-7).

MicroRNAs (miRNAs) are short, non-coding single-stranded RNAs, which have been identified as important post-transcriptional regulators. miRNAs specifically bind to the 3'-untranslational region (UTR) of target gene mRNAs by complementary base pairing in the RNA-induced silencing complex, and they degrade mRNA or repress the translation of target genes (8). miRNAs are essential for multiple biological processes, including cartilage formation and degeneration (9).

A previous study profiled the miRNA expression levels in chondrogenic human adipose-derived mesenchymal stem cells (hADSCs), and observed that the expression levels of miRNA-92a (miR-92a) were significantly altered (10). The present study hypothesized that miR-92a is involved in chondrogenesis and cartilage degeneration, and investigated the presence and biological function of miR-92a in chondrogenesis and cartilage degeneration. 


\section{Materials and methods}

The Ethics Committee of Sun Yat-Sen University (Guangzhou, China) approved the experiments performed in the present study. Procedures involving human subjects were performed in accordance with The Code of Ethics of the World Medical Association (Declaration of Helsinki). Informed consent was obtained from the patients prior to inclusion in the study. The experiments involving mice were performed in accordance with the Laboratory Animal Center of Sun Yat-Sen University and the Guide for the Care and Use of Laboratory Animals.

Primary chondrocyte isolation. Subsequent to obtaining informed consent, primary human chondrocytes (PHCs) were isolated from the cartilage of patients undergoing hip surgery. The patients included two females, aged 31 and 24 years, who were undergoing surgery for a femoral neck fracture at the First Affiliated Hospital of Sun Yat-Sen University. Patients with degraded cartilages, local or systemic immunological disorders or tumors were excluded from the investigation. The cartilage was carefully cut into sections and digested sequentially in pronase (cat. no. 10165921001; Roche Diagnostics, Basel, Switzerland) for 90 min and collagenase P (cat. no. 11213865001; Roche Diagnostics) for $\sim 7 \mathrm{~h}$ on a $37^{\circ} \mathrm{C}$ stirring-plate. The chondrocytes were then collected by centrifugation (1,000 $\mathrm{x} \mathrm{g}$ for $3 \mathrm{~min})$ of the digestion solution and then were rinsed with $\mathrm{Ca} / \mathrm{Mg}$-free phosphate-buffered saline (cat. no. 14190-094; Gibco Life Technologies, Paisley, UK) three times. The chondrocytes were seeded into flasks containing Dulbecco's modified Eagle's medium (DMEM/F12; cat. no. SH30023.01B; GE Healthcare Life Sciences, Logan, UT, USA) with 5\% fetal bovine serum (FBS; cat. no. 12657; Gibco Life Technologies), 2\% penicillin and streptomycin (cat. no. 15140-122; Gibco Life Technologies,) and ITS+ Premix (cat. no. 354352; BD Biosciences, Franklin Lakes, NJ, USA).

The isolation of primary mouse chondrocytes (PMCs) was performed, as previously reported (11). A total of 25 male mice were purchased from the Laboratory Animal Center of Sun Yat-Sen University (Guangzhou, China), and were maintained in five isolator cages in pathogen-free conditions. Mice were fed by the Laboratory Animal Center, and maintained at $18-22^{\circ} \mathrm{C}$ under $12 \mathrm{~h} \mathrm{light/dark}$ cycles, until the end of the experiments. The mice were washed with $70 \%$ ethanol prior to sacrifice. The mice were sacrificed via cervical dislocation under anesthesia with a sponge of $50 \mathrm{ml}$ anhydrous diethyl ether (Laboratory Animal Center of Sun Yat Sen University). Cartilage was carefully collected from the femoral head, femoral condyle and tibial plateau, and was digested in $3 \mathrm{mg} / \mathrm{ml}$ collagenase D (cat. no. 11088866001 ; Roche Diagnostics) at $37^{\circ} \mathrm{C}$ with agitation for $90 \mathrm{~min}$, followed by further digestion in collagenase D overnight. The cells were collected, filtered through a $48 \mu \mathrm{m}$ nylon mesh, and were then seeded into a culture flask at a density of 8,000 cells/ $\mathrm{cm}^{2}$ in M199 (Gibco Life Technologies; $10 \% \mathrm{FBS}, 1 \%$ penicillin and streptomycin).

Cell culture and chondrogenic differentiation. The hADSCs were purchased from Cyagen Bioinformatics (Suzhou), Inc. (cat. no. HUXMD-01001; Taicang, China). The hADSCs were maintained to permit expansion in DMEM (Cyagen Bioinformatics (Suzhou), Inc. 11965-092; Gibco Life
Technologies) with $10 \% \mathrm{FBS}, 100 \mu / \mathrm{ml}$ penicillin and $100 \mathrm{mg} / \mathrm{ml}$ streptomycin at $37^{\circ} \mathrm{C}$ in $5 \% \mathrm{CO}_{2}$ at saturated humidity. The cells were subcultured at $80 \%$ confluence and third generation cells were used for the subsequent characterization and chondrogenesis experiments.

The third generation hADSCs were harvested and resuspended in incomplete mesenchymal stem cell chondrogenic differentiation medium [cat. no. GUXMX-90041; Cyagen Bioinformatics (Suzhou), Inc.; $194 \mathrm{ml}$ basal medium, $20 \mu \mathrm{l}$ dexamethasone, $600 \mu \mathrm{l}$ ascorbic acid, $2 \mathrm{ml}$ ITS, $200 \mu \mathrm{l}$ sodium pyruvate, $200 \mu \mathrm{l}$ proline, $2 \mathrm{ml}$ transforming growth factor- $\beta 3$ ] at $2 \times 10^{7}$ cells $/ \mathrm{ml}$. Droplets $(12.5 \mu \mathrm{l})$ were then carefully added to each well of a 24-well plate. The hADSCs were allowed to adhere at $37^{\circ} \mathrm{C}$ for $90 \mathrm{~min}$, followed by the addition of $500 \mu 1$ chondrogenic differentiation medium $(10,12-14)$, which was replaced every 3 days.

ATDC5 mouse cells (Riken Cell Bank, Ibaraki, Japan) were cultured with DMEM/F12, 5\% FBS and $1 \%$ penicillin and streptomycin in a $37^{\circ} \mathrm{C}, 5 \% \mathrm{CO}_{2}$ humidified atmosphere. The culture medium was replaced every 2 days and the cells were subcultured when cells reached $90-100 \%$ confluence during the expansion culture. All the experiments were completed within 20 passages. The chondrogenic differentiation was induced using ITS+ Premix (15-17). The chondrogenic culture medium was then replaced daily.

PHCs were cultured in DMEM/F12, 5\% FBS, $1 \%$ penicillin and streptomycin and ITS+ at $37^{\circ} \mathrm{C}$ in a $5 \% \mathrm{CO}_{2}$ humidified atmosphere.

The PMCs were cultured for expansion in M199 (cat. no. 11150-059; Gibco Life Technologies), 10\% FBS, $1 \%$ penicillin and streptomycin, basic fibroblast growth factor (cat. no. 450-33; PeproTech, Oak Park, CA, USA) and epidermal growth factor (cat. no. $315-09$; PeproTech), at $37^{\circ} \mathrm{C}$ in a $5 \% \mathrm{CO}_{2}$ humidified atmosphere.

Interleukin-1 $\beta(I L-1 \beta)$-treated chondrocytes. ATDC5 cells were maintained in chondrogenic medium with $1 \%$ ITS+ for 14 days at $37^{\circ} \mathrm{C}$ to form chondrogenic ATDC5 cells. Chondrogenic ATDC5 cells, PHCs and PMCs, at the fourth passage, were treated with recombinant IL-1 $\beta$ (cat. no. 200-01B; PeproTech) at $1 \mathrm{ng} / \mathrm{ml}$ for $4 \mathrm{~h}(18-20)$.

Morphological analysis. The stained ATDC5 cells were fixed in formalin for $4 \mathrm{~h}$ at room temperature, and were stained with $1 \mathrm{mg} / \mathrm{ml}$ alcian blue $8 \mathrm{GX}$ for $20 \mathrm{~min}$ at room temperature, followed by examination using microscopy (Axio Imager Z1; Carl Zeiss AG, Oberkochen, Germany). The micromass was harvested at 0,7 and 14 days. The macromorphology was examined by imaging with the M205 FA microscope [Leica Microsystems AG, Heerbrugg, Switzerland]. The micromass was fixed in formalin, embedded in paraffin, and stained with alcian blue (14). Images were then captured under microscopy.

Reverse transcription-quantitative polymerase chain reaction (RT-qPCR) assays. Total RNA was extracted from the cells using an miRNeasy Mini kit (cat. no. 217004; Qiagen, Hilden, Germany), according to the manufacturer's instructions. The concentration and purity of the extracted RNA was analyzed using an Epoch Multi-Volume Spectrophotometer System (BioTek Instruments, Inc., Winooski, VT, USA). The cDNA of 
Table I. Primer sequences for reverse transcription-quantitative polymerase chain reaction.

Gene

$\mathrm{Mmu} / \mathrm{hsa}-\mathrm{U6}$

Mmu-GAPDH

Mmu/hsa-mir-92a

Mmu-col2a1

Mmu-Sox 9

Mmu-Col10a1

Mmu-Runx2

Mmu-mmp13

Mmu-TNF- $\alpha$

Hsa-GAPDH

Hsa-mmp13

Hsa-col2a1

Hsa-col10a1
Primer sequence (5'-3')

Forward: CTCGCTTCGGCAGCACA

Reverse: AACGCTTCACGAATTTGCGT

Forward: TGTGTCCGTCGTGGATCTGA

Reverse: TTGCTGTTGAAGTCGCAGGAG

TATTGCACTTGTCCCGGCCTG

Forward: CCCGCCTTCCCATTATTGAC

Reverse: GGGAGGACGGTTGGGTATCA

Forward: GGGGGTGAGCTTTGATTAATTC

Reverse: GGGATTTAAGGCTCAAGGTGTTT

Forward: TTCTGCTGCTAATGTTCTTGACC

Reverse: GGGATGAAGTATTGTGTCTTGGG

Forward: ATGCTTCATTCGCCTCACAAA

Reverse: GCACTCACTGACTCGGTTGG

Forward: ATGCATTCAGCTATCCTGGCCA

Reverse: AAGATTGCATTTCTCGGAGCCTG

Forward: GACGTGGAACTGGCAGAAGAG

Reverse: TTGGTGGTTTGTGAGTGTGAG

Forward: GGAGCGAGATCCCTCCAAAAT

Reverse: GGCTGTTGTCATACTTCTCATGG

Forward: TCCTGATGTGGGTGAATACAATG

Reverse: GCCATCGTGAAGTCTGGTAAAAT

Forward:GAGGGCAATAGCAGGTTCACGTA

Reverse: TGGGTGCAATGTCAATGATGG

Forward: CACCAGGCATTCCAGGATTCC

Reverse: AGGTTTGTTGGTCTGATAGCTC was obtained from mRNA and miRNAs using a PrimeScript ${ }^{\circledR}$ miRNA cDNA Synthesis kit (cat. no. DRR350; Takara Bio, Inc., Otsu, Japan), according to the manufacturer's instructions.

Semi-qPCR was performed using SYBR ${ }^{\circledR}$ Premix Ex Taq $^{\mathrm{TM}}$ II (cat. no. DRR081; Takara Bio, Inc.) and a Bio-Rad IQ5 system (Bio-Rad Laboratories, Inc., Hercules, CA, USA). The concentration of reagents and cycling conditions were according to the manufacturer's instructions. The cycles began at $95^{\circ} \mathrm{C}$ for $30 \mathrm{sec}$, followed by 40 cycles of $95^{\circ} \mathrm{C}$ for $5 \mathrm{sec}$ and $60^{\circ} \mathrm{C}$ for $30 \mathrm{sec}$. Ten nanograms of cDNA was added into the $25 \mu \mathrm{l}$ reaction volume. The primer sequences are presented in Table I. The reverse primer for the miRNAs was Uni-miR qPCR Primer (cat. no. D352; Takara Bio, Inc.). Quality control was performed by monitoring the melting curve. Fold differences in mRNA expression were calculated using the $\Delta \Delta \mathrm{Ct}$ method (21). All samples were measured in triplicate.

Transfection assays. The condition and efficiency of transfection assays were verified using a CY3-labelled siR-Ribo ${ }^{\mathrm{TM}}$ Transfection Control (cat. no. siN05815122149-1-1; Guangzhou RiboBio Co., Ltd., Guangzhou, China). The ATDC5 cells $\left(4 \times 10^{4}\right)$ were seeded into a 6-well plate with DMEM/F12 with $10 \% \mathrm{FBS}$, and were allowed to grow at $37^{\circ} \mathrm{C}$ until they had reached 50-70\% confluence. Lipofectamine ${ }^{\circledR} 2000$ transfection reagent (cat. no. 11668; Invitrogen Life Technologies, Carlsbad, CA, USA) was then used to transfect the micrON ${ }^{\mathrm{TM}}$ mmu-miR-92a-3p mimic/inhibitor (cat. nos. miR10000539-1-2 and miR20000539-1-2; Guangzhou RiboBio Co., Ltd.) and micrON ${ }^{\mathrm{TM}}$ mimic/inhibitor negative control (cat. no. miR01101-1-2 and miR02101-1-2; Guangzhou RiboBio Co., Ltd.) into the cells, according to the manufacturer's instructions. Subsequent to $6 \mathrm{~h}$ transfection, chondrogenic differentiation was induced by replacing the medium with chondrogenic medium containing $1 \%$ ITS+ Premix.

Target prediction. The potential target genes of miRNAs were predicted using the following online algorithms: miRanda (August 2010 release; http://www.microrna.org/), miRDB (MirTarget2_v4.0; http://www.mirdb.org/miRDB/), CLIP-Seq (2012-03-28; http://mirtarclip.mbc.nctu.edu.tw/) and TargetScan (version 6.2; http://targetscan.org/). Genes predicted by three or four separate algorithms were considered as potential target genes.

Based on these predicted target genes, the signaling pathways potentially regulated by miR-92a were analyzed using the Kyoto Encyclopedia of Genes and Genomes (KEGG, kobas2.0-20120208; http://www.genome.jp/kegg/) database and the possible function of miR-92a was predicted. 

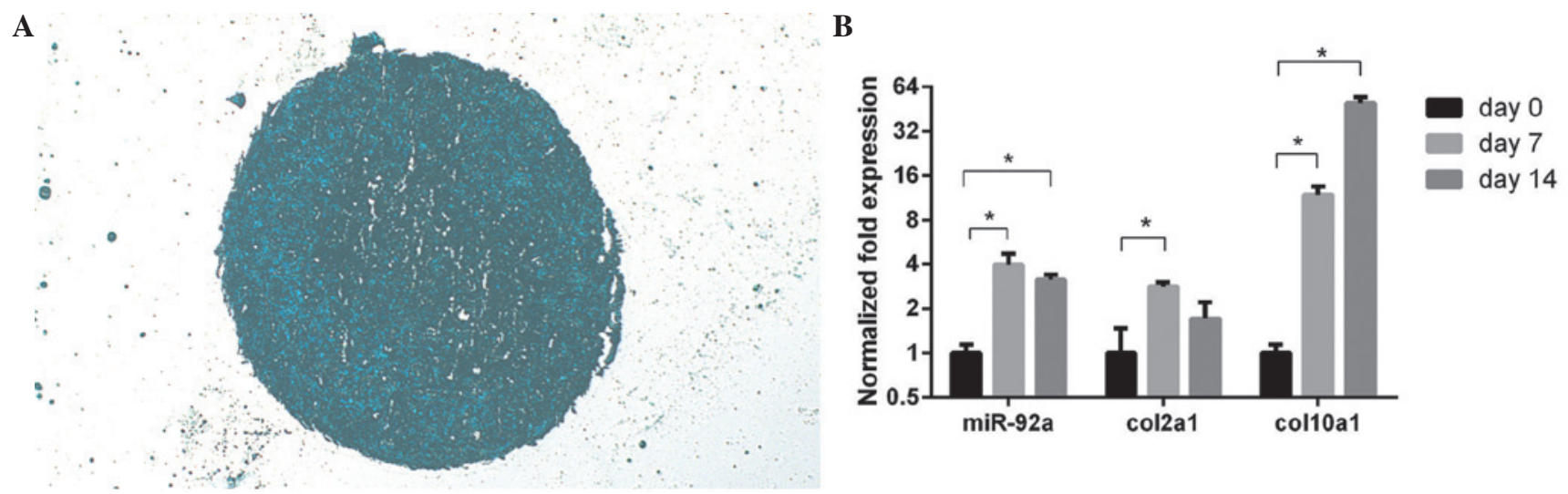

Figure 1. Chondrogenic adipose-derived mesenchymal stem cell micromass and the expression of miR-92a. (A) Subsequent to chondrogenesis for 7 days, the micromass was embedded in paraffin and cut into sections, followed by alcian blue staining (magnification, x100). (B) RNA was isolated from the micromass and the expression levels of miR-92a, col2a1, and col10a1 were examined using reverse transcription-quantitative polymerase chain reaction. Error bars indicate the mean \pm standard deviation. $\mathrm{P}<0.05$. miR-92a, microRNA-92a.

A

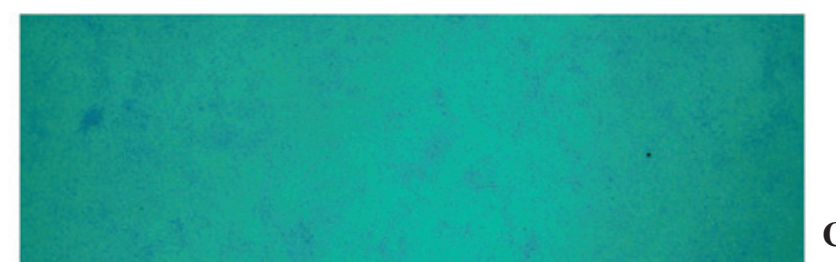

C

B
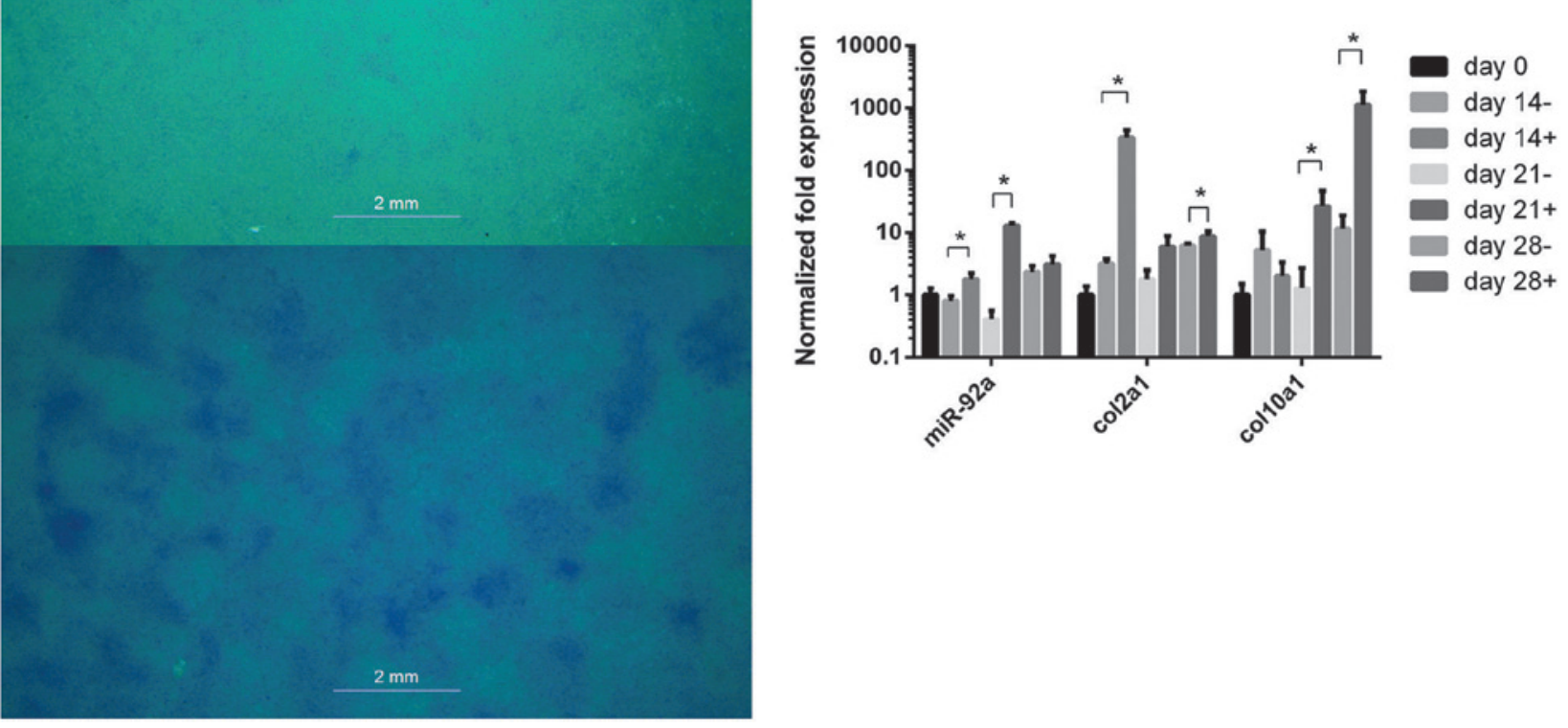

Figure 2. Chondrogenic ATDC5 and miR-92a expression. Chondrogenic ATDC5 cells were induced with ITS+ Premix, fixed with formalin and stained with alcian blue. (A) ATDC5 without ITS+ Premix exhibited low levels of alcian blue staining. (B) Chondrogenic ATDC5 cells exhibited more marked staining. (C) Expression levels of miR-92a, col2a1 and col10a1 in chondrogenic ATDC5 cells, examined using reverse transcription-quantitative polymerase chain reaction, compared with the negative control of ATDC5 without ITS+ Premix. Error bars indicate the mean \pm standard deviation. "P<0.05. miR-92a, microRNA-92a.

Statistical analysis. All experiments were performed in triplicate. The quantitative data was expressed as the mean \pm confidence interval (mean $\pm 1 / 2 \mathrm{CI})$. Differences between the groups were analyzed using Student's t-text or analysis of variance with SPSS, version 13.0 (SPSS, Inc., Chicago, IL, USA). The least significant difference test and Tamhane's T2 test were used in conditions with, and without, equal variances, respectively. The qualitative data was analyzed using Fisher's Exact test. $\mathrm{P}<0.05$ was considered to indicate a statistically significant difference.

\section{Results}

Expression of miR-92a is elevated in chondrogenic differentiation. The micromass produced from the hADSCs in chondrogenic medium was embedded in paraffin, cut into sections and stained with alcian blue (Fig. 1A). The expression levels of miR-92a, col2a1 and col10al increased in the chondrogenic hADSCs cells (Fig. 1B). The expression levels of mir-92a and the chondrogenic marker of col2al peaked at day 7 of chondrogenic induction, and the hypertrophic marker of col10al peaked at day 14. 
Table II. Relative mRNA expression levels in ATDC5 cells transfected with miR-92a mimic or inhibitor.

\begin{tabular}{|c|c|c|c|c|c|c|c|c|c|c|c|c|}
\hline \multirow[b]{2}{*}{ Gene } & \multicolumn{3}{|c|}{$\operatorname{Mimic}(50 \mathrm{nM})$} & \multicolumn{3}{|c|}{$\operatorname{Mimic}(100 \mathrm{nM})$} & \multicolumn{3}{|c|}{ Inhibitor (50 nM) } & \multicolumn{3}{|c|}{ Inhibitor (100 nM) } \\
\hline & $\begin{array}{l}\text { Fold } \\
\text { change }\end{array}$ & P-value & SD & $\begin{array}{l}\text { Fold } \\
\text { change }\end{array}$ & P-value & SD & $\begin{array}{l}\text { Fold } \\
\text { change }\end{array}$ & P-value & SD & $\begin{array}{l}\text { Fold } \\
\text { change }\end{array}$ & P-value & SD \\
\hline Col2a1 & 0.89 & 0.24 & 0.14 & 0.74 & 0.02 & 0.10 & 1.11 & 0.14 & 0.10 & 0.40 & $<0.001$ & 0.10 \\
\hline Col10a1 & 1.10 & 0.17 & 0.06 & 1.11 & 0.12 & 0.01 & 1.36 & $<0.001$ & 0.12 & 0.75 & 0.001 & 0.05 \\
\hline Comp & 0.61 & $<0.001$ & 0.07 & 1.24 & 0.001 & 0.07 & 0.91 & 0.014 & 0.06 & 0.24 & $<0.001$ & 0.04 \\
\hline $\mathrm{Agc}$ & 3.52 & 0.001 & 0.62 & 6.89 & $<0.001$ & 0.76 & 0.56 & $<0.001$ & 0.01 & 0.26 & $<0.001$ & 0.01 \\
\hline Mmp-13 & 1.94 & 0.004 & 0.47 & 3.03 & $<0.001$ & 0.14 & 1.00 & 0.994 & 0.03 & 0.67 & $<0.001$ & 0.02 \\
\hline Col9a2 & 3.80 & 0.01 & 0.60 & 14.97 & $<0.001$ & 1.95 & 0.37 & $<0.001$ & 0.09 & 0.10 & $<0.001$ & 0.02 \\
\hline Sox9 & 2.45 & $<0.001$ & 0.26 & 4.15 & $<0.001$ & 0.50 & 1.18 & 0.15 & 0.16 & 1.10 & 0.40 & 0.23 \\
\hline Runx2 & 1.59 & $<0.001$ & 0.16 & 2.16 & $<0.001$ & 0.12 & 0.91 & 0.19 & 0.02 & 1.00 & 0.98 & 0.13 \\
\hline
\end{tabular}

Expression of chondrogenic markers in ATDC5 cells transfected with miR-92a mimic or inhibitor at the indicated doses. Subsequent to transfection, ATDC5 cells were cultured in chondrogenic medium with ITS+ Premix for 4 days. Chondrogenic markers were measured using reverse transcription-quantitative polymerase chain reaction. Co19a2 and aggrecan were markedly upregulated in the miR-92a mimic groups, but were downregulated in the inhibitor groups, in a dose-dependent manner. No clear trends were observed for the other markers. miR-92a, microRNA-92a; SD, standard deviation.

A

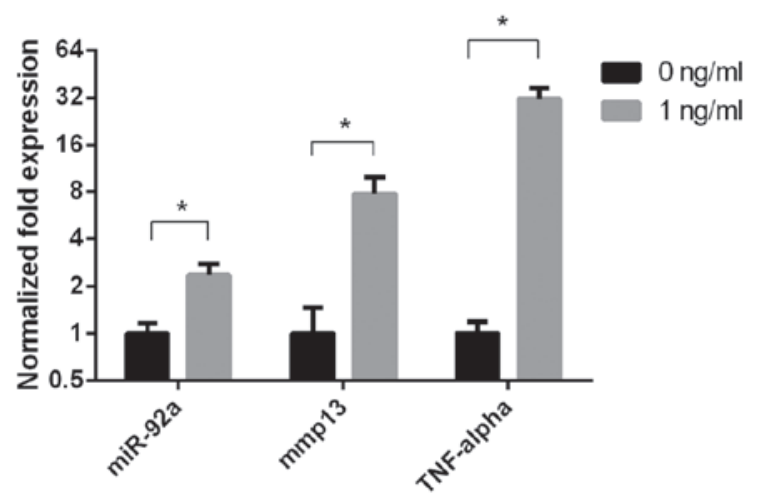

C
B

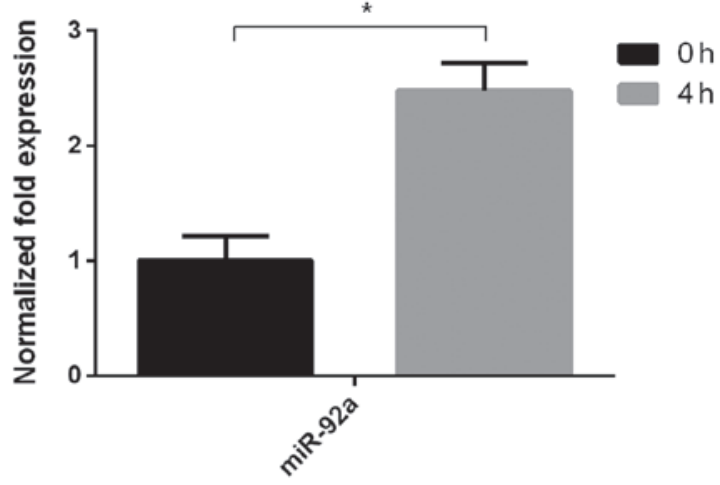

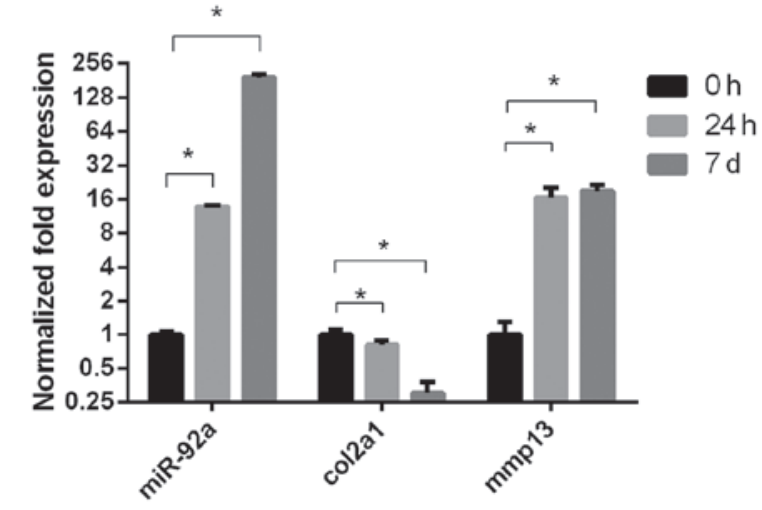

Figure 3. Experssion of miR-92a in chondrocytes with IL-1 $\beta$. Error bars indicate the mean \pm standard deviation. (A) Expression of miR-92a was elevated in primary mouse chondrocytes treated with $1 \mathrm{ng} / \mathrm{ml} \mathrm{IL-1 \beta}$ for $4 \mathrm{~h}$. (B) Expression of miR-92a was elevated in primary human chondrocytes treated with $1 \mathrm{ng} / \mathrm{ml}$ IL-1 $\beta$ for $4 \mathrm{~h}$. No mmp13 was detected in the control group. (C) Expression of miR-92a was elevated in a time-dependent manner in the chondrogenic ATDC5 cells treated with $1 \mathrm{ng} / \mathrm{ml}$ IL-1 $\beta$. miR-92a, microRNA-92a; IL-1 $\beta$, interleukin-1 $\beta$.

Following 14 days of chondrogenic differentiation with ITS+ Premix, ATDC5 cells exhibited marked staining with alcian blue, compared with the cells without ITS+ Premix (Fig. 2A and B). The expression of col2al peaked at day 14 , col10a1 peaked at day 28 and miR-92a peaked at day 21 (Fig. 2C).
Expression of miR-92a is increased in $I L-1 \beta$-treated chondrocytes. Expression levels of miR-92a and mmp13 were upregulated in the PHCs and PMCs treated with $1 \mathrm{ng} / \mathrm{ml} \mathrm{IL-1 \beta}$ for $4 \mathrm{~h}$, compared with the control (Fig. 3A and B). The expression levels of miR-92a and mmp13 were elevated, and that of col2a1 was suppressed in a time-dependent manner 


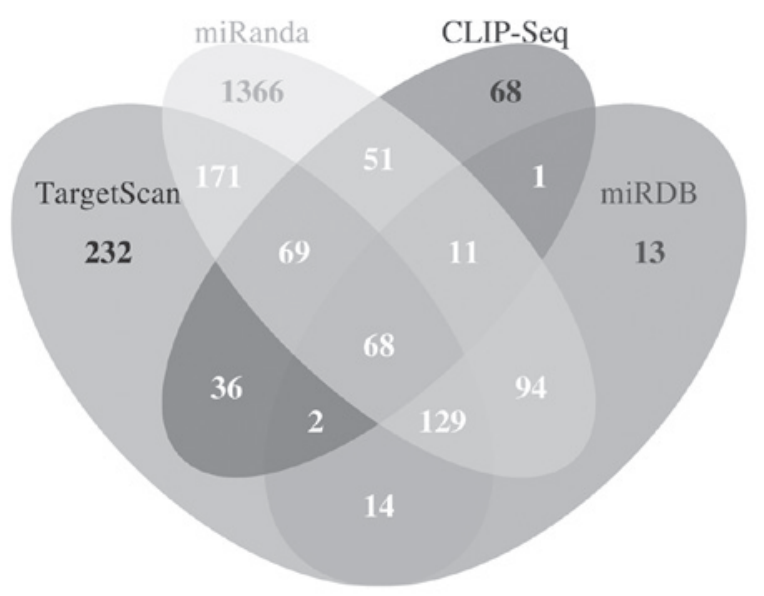

Figure 4. Potential target genes of miR-92a, predicted using four algorithms. In order to minimize possible false positive prediction, only genes predicted by three or four algorithms were identified as potential target genes of miR-92a. miR-92a, microRNA-92a. Numbers refer to genes predicted by one of the four software program.

in the chondrogenic ATDC5 cells treated with $1 \mathrm{ng} / \mathrm{ml} \mathrm{IL-1 \beta}$ (Fig. 3C)

Col9a 2 and aggrecan may be regulated by $m i R$-92a. In order to investigate the effects of miR-92a on chondrogenesis, the expression levels of miR-92a were manipulated via transfection with a mimic or inhibitor. The altered expression of miR-92a affected chondrogenic markers, increasing the expression levels of col9a2 and aggrecan with the miR-92a mimic in a dose-dependent manner, compared with the untransfected control. In addition, the miR-193b-3p inhibitor reduced the expression levels of col9a 2 and aggrecan in a dose-dependent manner, compared with the control (Table II).

Predicted target genes, signaling pathways and functions of $m i R-92 a$. The four algorithms, miRanda, miRDB, CLIP-Seq and TargetScan, were used for prediction of the miR-92a target genes. The general distribution of the predicted potential target genes is shown in Fig. 4. A total of 279 genes were predicted by three or four algorithms and were considered as potential target genes of miR-92a.

In addition to RT-qPCR, KEGG analysis was used to investigate the signaling pathways by which miR-92a may regulate chondrogenesis, based on the predicted potential target genes. The potential target genes were clustered based on their involvement in signaling pathways, and signaling pathways, which have been previously identified to be involved in chondrogenesis or cartilage degeneration were selected (5,22-25). The P-value was determined based on the number of potential target genes and the number of total genes in each signaling pathway. Of all of the predicted signaling pathways, the PI3K-Akt $(\mathrm{P}=0.064)$, ErbB $(\mathrm{P}=0.076)$ and focal adhesion kinase pathways $(\mathrm{P}=0.014)$, ECM-receptor interaction $(\mathrm{P}=0.024)$ and the mTOR signaling pathway $(\mathrm{P}=0.007)$ were identified as significant (Table III, Fig. 5). 
A

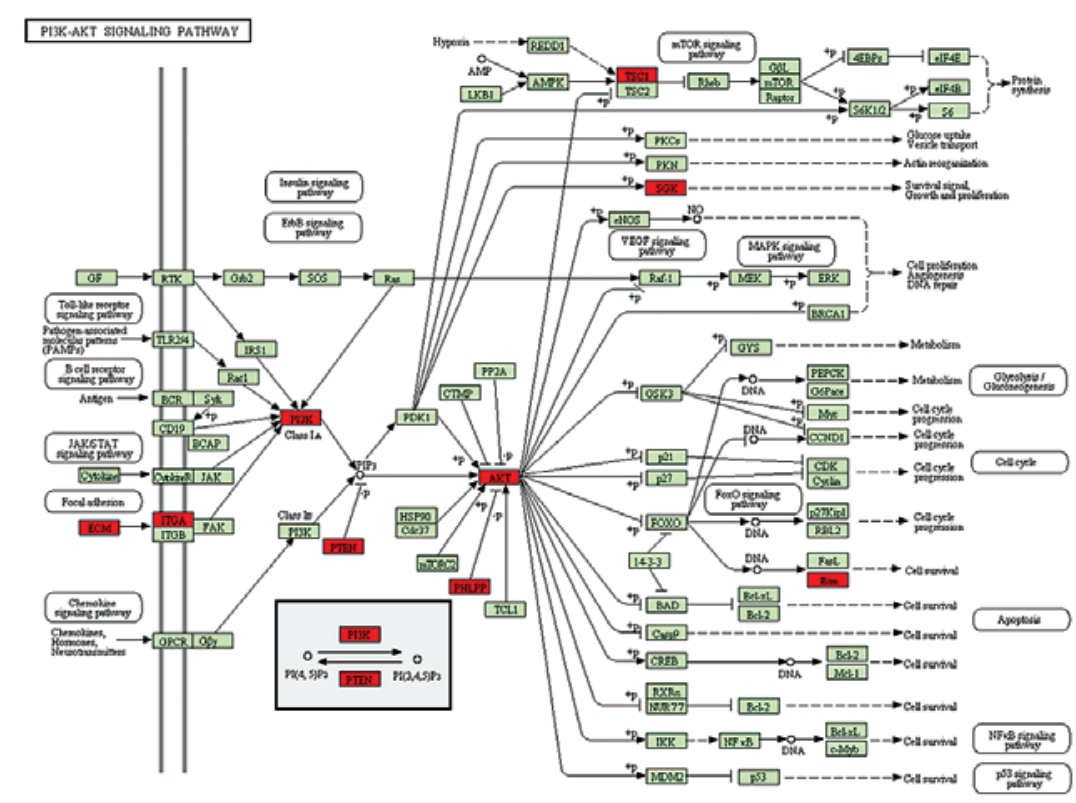

B

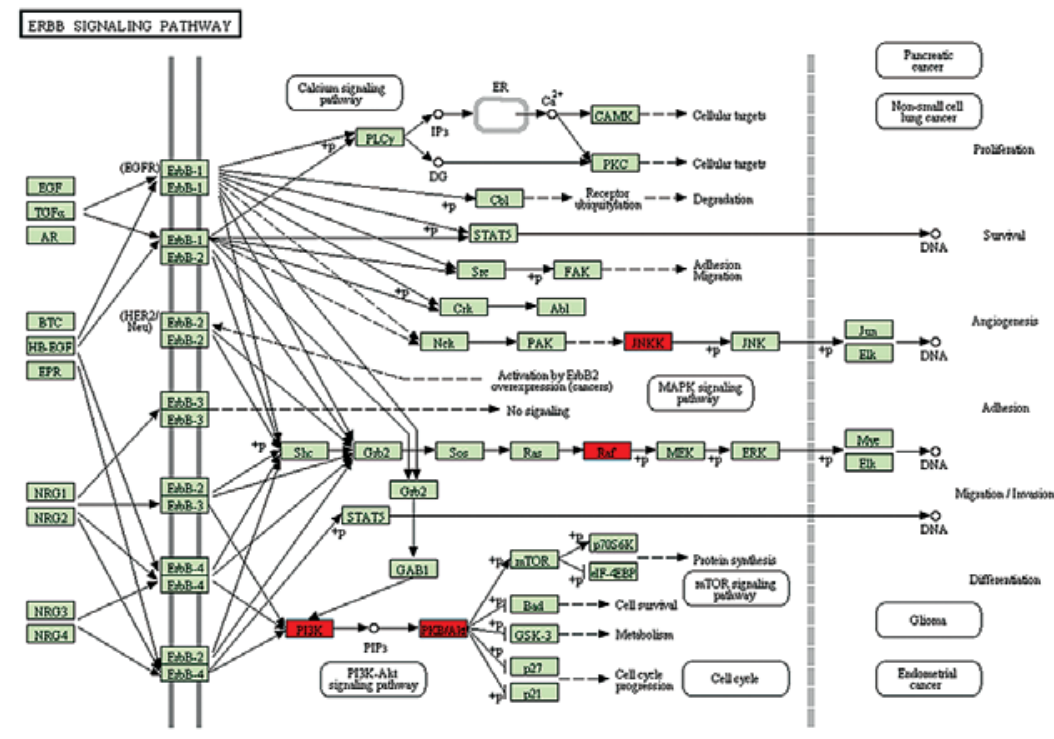

C

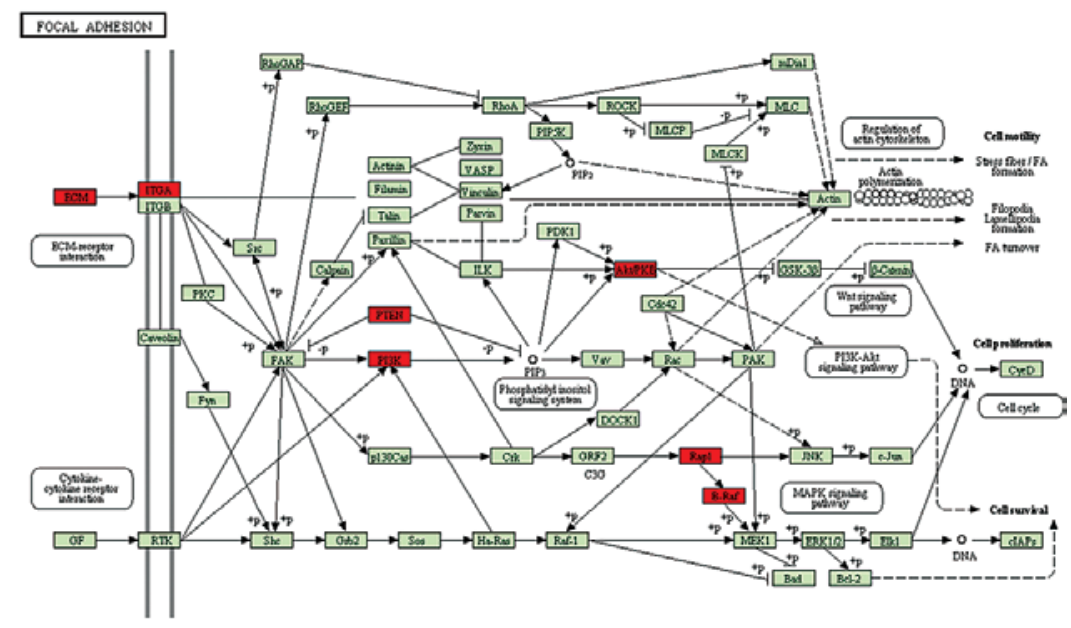

Figure 5. Predicted signaling pathways mediating the effects of miR-92a on chondrogenesis and cartilage degeneration. Predicted potential target genes of miR-92a are indicated by the red boxes. (A) PI3K-Akt, (B) ErbB and (C) focal adhesion signaling pathways were predicted based on the potential target genes of miR-92a. miR-92a, microRNA-92a; PI3K, phosphoinositide-3 kinase; mTOR, mammalian target of rapamycin. 
D

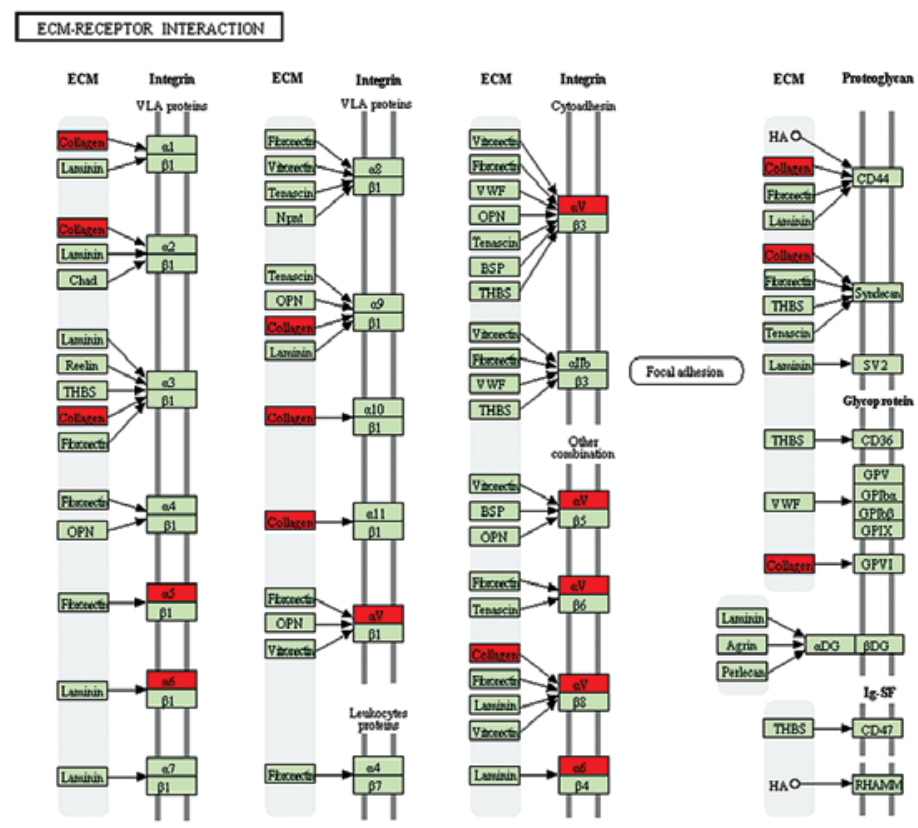

$\mathbf{E}$

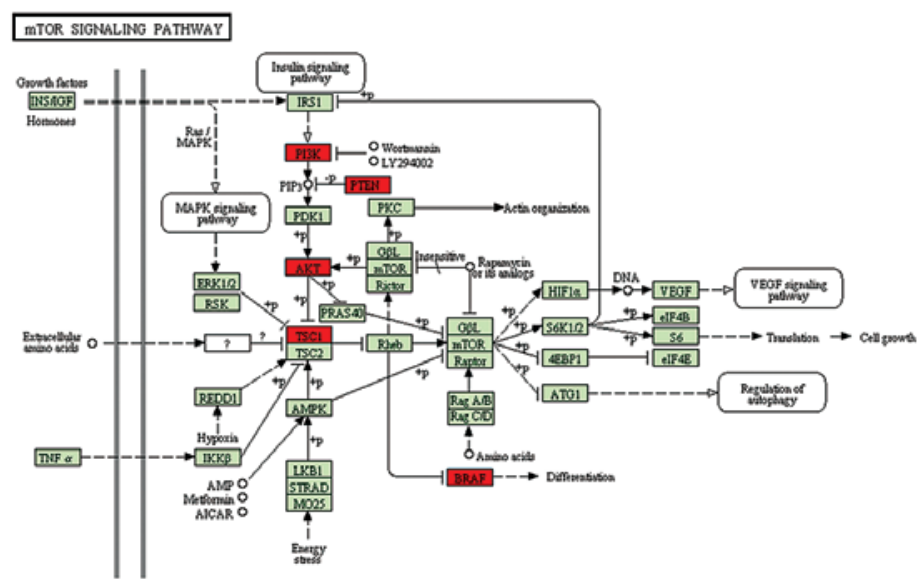

Figure 5. Continued. Predicted signaling pathways mediating the effects of miR-92a on chondrogenesis and cartilage degeneration. Predicted potential target genes of miR-92a are indicated by the red boxes. (D) ECM-receptor interaction and (E) mTOR signaling pathways were predicted based on the potential target genes of miR-92a. miR-92a, microRNA-92a; PI3K, phosphoinositide-3 kinase; mTOR, mammalian target of rapamycin.

\section{Discussion}

Previous studies have suggested a role for miR-92a in renal tumorigenesis via the gene expression of VHL (26), and in human acute promyelocytic leukemia via the expression of p63 (27). An additional study identified the positive effects of miR-92a on the proliferation, differentiation and survival of chondrogenic progenitors via the targeting of nog3, an inhibitor of the bone morphogenetic protein (BMP) signaling pathway (28). Although miR-92a was observed to contribute to chondrogenesis by enhancing the expression of col2al in the study by Ning et al (28), no significant trend in the expression of col2al was not observed in the present study following transfection of either the miR-92a mimic or inhibitor. This discrepancy may be due to differences in experimental subjects and signaling pathways. In the study by Ning et al (28), the BMP signaling pathway (smad1/5/8) and the inhibitor of BMP pathway (nog3) were observed to mediate the effects of
miR-92a on in vivo pharyngeal chondrogenesis. In the present study, cultured ATDC5 cells were used for the investigation of miR-92a and chondrogenesis, which are associated with the autocrine transforming growth factor- $\beta(\operatorname{smad} 2 / 3)$ signaling pathway (29).

In the study by Ning et al (28), the morphological defects resulting from the inhibition of nog 3 , one of the target genes of miR-92a's, were partially reversed by p53 co-inhibition, suggesting a contribution of miR-92a-nog3-apoptosis/proliferation to in vivo morphological regulation of pharyngeal cartilage formation. During chondrogenesis, high levels of type 9 collagen and aggrecan are expressed, along with additional matrix proteins to form the cartilage matrix, with col9a2 and aggrecan considered as chondrogenic markers (30). Col9a2 and aggrecan were previously demonstrated to be associated with a number of diseases, including osteoarthritis $(31,32)$, degeneration of intervertebral discs $(33,34)$ and multiple epiphyseal dysplasia, characterized as the deformed deposition 
of cartilage at the ends of the bones $(32,35,36)$. The present study hypothesized that col9a 2 may be another mediator of the degeneration of cartilage, followed by miR-92a knockdown. For the upstream regulation of col9a2 and aggrecan, Sox 9 has been previously suggested as to be critical in initiating the expression of col9a2 and aggrecan (37), although multiple enhancers have been observed to initiate expression of aggrecan (38). However, more detailed information is required on the regulation of the expression levels of col9a2 and aggrecan in order to identify the cure for these diseases. In the present study, the results indicated that miR-92a may contribute to the upregulation of col9a 2 and aggrecan, without enhancing the expression of sox 9 . These results provided novel insight into the upstream regulation of col9a2 and aggrecan, beyond what is already known about sox 9 in relation to col9a2 and aggrecan. In addition, the results suggested another possible mechanism of a miR-92a-col9a2-cartilage deformity axis contributing to cartilage deformity following miR-92a knockdown. Further investigations are required in order to verify the effect of miR-92a on the in vivo expression levels of col9a2, aggrecan, and cartilage degeneration, and to determine the underlying mechanisms.

Several previous studies investigating miRNAs used one or two algorithms to predict the target genes, with subsequent mechanistic experiments, based on the predicted genes $(39,40)$. However, each of these widely used algorithms has an intrinsic false positive rate. The false positive rate is 22-31\% for TargetScan, 24-39\% for miRanda and $30 \%$ for PicTar (41). In the present study, four algorithms were used, and an intersection set of predicted genes from at least three algorithms was identified as a potential target gene. Based on the potential target genes, KEGG analysis was then used to predict several signaling pathways that possibly contribute to the effect of miR-92a on chondrogenesis. KEGG is a database, which is usually used for the prediction of function and signaling pathways from large scale molecular information of high-throughput experiments, including sequencing. This prediction method enables the minimization of false positive rates and assist in understanding the possible function of miR-92a in a wider context (42). Investigations of underlying mechanisms can be performed using these predictions, including luciferase reporter assays of miR-92a and 3'-UTR of Akt1.

In conclusion, the present study demonstrated the presence of miR-92a in chondrogenesis and the chondrocyte response induced by IL-1 $\beta$. The positive contribution of miR-92a in the expression of col9a2 and aggrecan was observed and the PI3K-Akt, ErbB and focal adhesion kinase pathways, ECM-receptor interaction, and mTOR signaling pathway were indicated as potential mediators of the effects of miR-92a on chondrogenesis and cartilage degeneration.

\section{Acknowledgements}

The authors would like to thank to Dr Xuerong Li, Dr Shan Li and Dr Shang Mei at the Department of Parasitology, Zhongshan School of Medicine, Sun Yat-sen University (Guangzhou, China) for their technical assistance.

The present study was supported by the National Natural Science Foundation of China (grant nos. 81301558, 81371941 and 81171709), the Doctoral Scientific Fund Project of the Ministry of Education of China (grant no. 20130171120074) and the Natural Science Foundation of Guangdong Province, China (grant no. s2013040016269). The sponsors had no involvement in the study design; collection, analysis and interpretation of data; the writing of the manuscript; or in the decision to submit the manuscript for publication.

\section{References}

1. Li Y, Wei X, Zhou J and Wei L: The age-related changes in cartilage and osteoarthritis. Biomed Res Int 2013: 916530, 2013.

2. Mathieu PS and Loboa EG: Cytoskeletal and focal adhesion influences on mesenchymal stem cell shape, mechanical properties and differentiation down osteogenic, adipogenic and chondrogenic pathways. Tissue Eng Part B Rev 18: 436-444, 2012.

3. Beier F and Loeser RF: Biology and pathology of Rho GTPase, PI-3 kinase-Akt and MAP kinase signaling pathways in chondrocytes. J Cell Biochem 110: 573-580, 2010.

4. Chen J, Crawford R and Xiao Y: Vertical inhibition of the $\mathrm{PI} 3 \mathrm{~K} / \mathrm{Akt} / \mathrm{mTOR}$ pathway for the treatment of osteoarthritis. J Cell Biochem 114: 245-249, 2013.

5. Rokutanda S, Fujita T, Kanatani N, Yoshida CA, Komori H, Liu W, Mizuno A and Komori T: Akt regulates skeletal development through GSK3, mTOR and FoxOs. Dev Biol 328: 78-93, 2009.

6. Malemud CJ: Intracellular signaling pathways in rheumatoid arthritis. J Clin Cell Immunol 4: 160, 2013.

7. Xian CJ: Roles of epidermal growth factor family in the regulation of postnatal somatic growth. Endocr Rev 28: 284-296, 2007.

8. Carthew RW and Sontheimer EJ: Origins and Mechanisms of miRNAs and siRNAs. Cell 136: 642-655, 2009.

9. Hong E and Reddi AH: MicroRNAs in chondrogenesis, articular cartilage and osteoarthritis: Implications for tissue engineering. Tissue Eng Part B Rev 18: 445-453, 2012.

10. Zhang Z, Kang Y, Zhang Z, Zhang H, Duan X, Liu J, Li X and Liao W: Expression of microRNAs during chondrogenesis of human adipose-derived stem cells. Osteoarthritis Cartilage 20: 1638-1646, 2012

11. Thirion S and Berenbaum F: Culture and phenotyping of chondrocytes in primary culture. Methods Mol Med 100: 1-14, 2004.

12. Zhang ZJ, Zhang H, Kang Y, Sheng PY, Ma YC, Yang ZB, Zhang ZQ, Fu M, He AS, Liao WM, et al: miRNA expression profile during osteogenic differentiation of human adipose-derived stem cells. J Cell Biochem 113: 888-898, 2012.

13. Zhang L, Su P, Xu C, Yang J, Yu W and Huang D: Chondrogenic differentiation of human mesenchymal stem cells: A comparison between micromass and pellet culture systems. Biotechnol Lett 32: 1339-1346, 2010.

14. Estes BT, Diekman BO, Gimble JM and Guilak F: Isolation of adipose-derived stem cells and their induction to a chondrogenic phenotype. Nat Protoc 5: 1294-1311, 2010.

15. Yao Y and Wang Y: ATDC5: An excellent in vitro model cell line for skeletal development. J Cell Biochem 114: 1223-1229. 2013.

16. Newton PT, Staines KA, Spevak L, Boskey AL, Teixeira CC, Macrae VE, Canfield AE and Farquharson C: Chondrogenic ATDC5 cells: An optimised model for rapid and physiological matrix mineralisation. Int J Mol Med 30: 1187-1193, 2012.

17. Atsumi T, Miwa Y, Kimata K and Ikawa Y: A chondrogenic cell line derived from a differentiating culture of AT805 teratocarcinoma cells. Cell Differ Dev 30: 109-116, 1990.

18. Miyaki S, Nakasa T, Otsuki S, Grogan SP, Higashiyama R, Inoue A, Kato Y, Sato T, Lotz MK, Asahara $\mathrm{H}$, et al: MicroRNA-140 is expressed in differentiated human articular chondrocytes and modulates interleukin-1 responses. Arthritis Rheum 60: 2723-2730, 2009.

19. Simsa-Maziel S and Monsonego-Ornan E: Interleukin-1 $\beta$ promotes proliferation and inhibits differentiation of chondrocytes through a mechanism involving down-regulation of FGFR-3 and p21. Endocrinology 153: 2296-2310, 2012.

20. MacRae VE, Farquharson $C$ and Ahmed SF: The restricted potential for recovery of growth plate chondrogenesis and longitudinal bone growth following exposure to pro-inflammatory cytokines. J Endocrinol 189: 319-328, 2006.

21. Schmittgen TD and Livak KJ: Analyzing real-time PCR data by the comparative C(T) method. Nat Protoc 3: 1101-1108, 2008. 
22. Kita K, Kimura T, Nakamura N, Yoshikawa H and Nakano T: PI3K/Akt signaling as a key regulatory pathway for chondrocyte terminal differentiation. Genes Cells 13: 839-850, 2008

23. Fisher MC, Clinton GM, Maihle NJ, Dealy CN: Requirement for ErbB2/ErbB signaling in developing cartilage and bone. Dev Growth Differ 49: 503-513, 2007.

24. Takahashi I, Onodera K, Sasano Y, et al: Effect of stretching on gene expression of betal integrin and focal adhesion kinase and on chondrogenesis through cell-extracellular matrix interactions. Eur J Cell Biol 82: 182-192, 2003.

25. Knudson CB: Hyaluronan and CD44: strategic players for cell-matrix interactions during chondrogenesis and matrix assembly. Birth Defects Res C Embryo Today 69: 174-196, 2003.

26. Valera VA, Walter BA, Linehan WM and Merino MJ: Regulatory Effects of microRNA-92 (miR-92) on VHL Gene Expression and the Hypoxic Activation of miR-210 in clear cel renal cell carcinoma. J Cancer 2: 515-526, 2011.

27. Sharifi M, Salehi R, Gheisari Y and Kazemi M: Inhibition of microRNA miR-92a induces apoptosis and inhibits cell proliferation in human acute promyelocytic leukemia through modulation of p63 expression. Mol Biol Rep 41: 2799-2808, 2014.

28. Ning G, Liu X, Dai M, Meng A and Wang Q: MicroRNA-92a upholds Bmp signaling by targeting noggin 3 during pharyngeal cartilage formation. Dev Cell 24: 283-295, 2013.

29. Kawai J, Akiyama H, Shigeno C, Ito H, Konishi J and Nakamura T: Effects of transforming growth factor-beta signaling on chondrogenesis in mouse chondrogenic EC cells, ATDC5. Eur J Cell Biol 78: 707-714, 1999.

30. Okazaki K and Sandell LJ: Extracellular matrix gene regulation. Clin Orthop Relat Res (427 Suppl): S123-S128, 2004.

31. Nakki A, Videman T, Kujala UM, Suhonen M, Männikkö M, Peltonen L, Battié MC, Kaprio J and Saarela J: Candidate gene association study of magnetic resonance imaging-based hip osteoarthritis (OA): Evidence for COL9A2 gene as a common predisposing factor for hip OA and lumbar disc degeneration. J Rheumatol 38: 747-752, 2011.
32. Gleghorn L, Ramesar R, Beighton $\mathrm{P}$ and Wallis G: A mutation in the variable repeat region of the aggrecan gene (AGC1) causes a form of spondyloepiphyseal dysplasia associated with severe, premature osteoarthritis. Am J Hum Genet 77: 484-490, 2005.

33. Aladin DM, Cheung KM, Chan D, Yee AF, Jim JJ, Luk KD and Lu WW: Expression of the Trp2 allele of COL9A2 is associated with alterations in the mechanical properties of human intervertebral discs. Spine (Phila Pa 1976) 32: 2820-2826, 2007.

34. Kim NK, Shin DA, Han IB, Yoo EH, Kim SH and Chung SS: The association of aggrecan gene polymorphism with the risk of intervertebral disc degeneration. Acta Neurochir (Wien) 153: 129-133, 2011.

35. Fiedler J, Stöve J, Heber F and Brenner RE: Clinical phenotype and molecular diagnosis of multiple epiphyseal dysplasia with relative hip sparing during childhood (EDM2). Am J Med Genet 112: 144-153, 2002.

36. Briggs MD, Choi H, Warman ML, Loughlin JA, Wordsworth $\mathrm{P}$, Sykes BC, Irven CM, Smith M, Wynne-Davies R, Lipson MH, et al: Genetic mapping of a locus for multiple epiphyseal dysplasia (EDM2) to a region of chromosome 1 containing a type IX collagen gene. Am J Hum Genet 55: 678-684, 1994.

37. Bi W, Deng JM, Zhang Z, Behringer RR and de Crombrugghe B: Sox9 is required for cartilage formation. Nat Genet 22: 85-89, 1999.

38. Hu G, Codina M and Fisher S: Multiple enhancers associated with ACAN suggest highly redundant transcriptional regulation in cartilage. Matrix Biol 31: 328-337, 2012.

39. Ge YZ, Xu LW, Xu Z, et al: Expression Profiles and Clinical Significance of MicroRNAs in Papillary Renal Cell Carcinoma: A STROBE-Compliant Observational Study. Medicine (Baltimore) 94: e767, 2015.

40. Xie J, Tan ZH, Tang X, et al: MiR-374b-5p suppresses RECK expression and promotes gastric cancer cell invasion and metastasis. World J Gastroenterol 20: 17439-17447, 2014.

41. Bentwich I: Prediction and validation of microRNAs and their targets. Febs Lett 579: 5904-5910, 2005.

42. ElHefnawi M, Soliman B, Abu-Shahba N and Amer M: An integrative meta-analysis of microRNAs in hepatocellular carcinoma. Genomics Proteomics Bioinformatics 11: 354-367, 2013. 